\title{
Long-term monitoring of classical swine fever in wild boar (Sus scrofa sp.) using serological data
}

\author{
Sophie Rossi ${ }^{\mathrm{a}, \mathrm{b}}$, Marc ARTOIS $^{\mathrm{a}}$, Dominique PONTIER ${ }^{\mathrm{c}}$, \\ Catherine CRUCIÈRE ${ }^{\mathrm{d}}$, Jean HARS ${ }^{\mathrm{e}}$, Jacques BARRAT $^{\mathrm{f}}$, Xavier PACHOLEK $^{\mathrm{b}}$, \\ Emmanuelle FROMONT ${ }^{\mathrm{c} *}$
}

\author{
a École Nationale Vétérinaire de Lyon, Unité Microbiologie, Pathologie infectieuse et Épidémiologie, \\ 1 avenue Bourgelat, BP 83, 69280 Marcy-l'Étoile, France \\ b Direction Générale de l'Alimentation, Ministère de l'Agriculture, de l'Alimentation, \\ de la Pêche et des Affaires Rurales, 251 rue de Vaugirard, 75732 Paris Cedex 15, France \\ c UMR 5558, Biométrie et Biologie Évolutive, Bâtiment Mendel, Université Claude Bernard Lyon 1, \\ 43 Bd du 11 novembre 1918, 69622 Villeurbanne Cedex, France \\ d Agence Française de Sécurité Sanitaire des Aliments (AFSSA), 23 avenue du Général De Gaulle, \\ 94706 Maisons-Alfort Cedex, France \\ e Office National de la Chasse et de la Faune Sauvage, 5 allée de Bethléem, \\ ZI de Mayencin, 38610 Gières, France \\ f Agence Française de Sécurité Sanitaire des Aliments (AFSSA), \\ BP 9, Domaine de Pixérécourt, 54220 Malzéville, France
}

(Received 12 March 2004; accepted 30 June 2004)

\begin{abstract}
In the European Community, epizootics of classical swine fever (CSF) in the wild boar (Sus scrofa) are compulsorily monitored because transmission may occur between wild boars and domestic pigs, causing heavy economic losses to the pork industry. The estimation of incidence in populations of wild boars is generally based on viroprevalence. However, viral isolation becomes rare when the incidence is low because the virus cannot be detected for more than a few weeks following infection. On the contrary, seroprevalence is detectable at low incidence levels, because antibodies can be detected for the lifetime of the infected animal. We thus attempted to analyse the long-term evolution of CSF incidence using serological data. The data came from France, where CSF had been monitored from 1992 to 2002, and where the virus has not been detected since 1997. We assumed that the overall seroprevalence would estimate the proportion of immune wild boars, that seroprevalence in juveniles would approximate incidence and that seroprevalence in different age classes would show the evolution of incidence in a given cohort. Spatial and temporal trends of incidence and seroprevalence were explored using logistic modelling and the spatial trend was analysed using polynomial regression. In 1992, incidence peaked in the northern area. After 1993, incidence decreased but remained the highest in the northern area. After 2000, no seropositive juvenile was observed, suggesting the extinction of the epizootic. Our results support the reliability of serological monitoring since it allowed a longer detection of viral transmission and provided more information on the spatio-temporal evolution of incidence than did viral isolation. We advocate that the highest persistence of infection in northeastern France is not independent from infection persistence in Reinland-Pfalz (Germany). Such persistence may be due to favourable local conditions and/or the social organisation of wild boars.
\end{abstract}

\section{classical swine fever / wild boar / spatial / monitoring / serology}

* Corresponding author: fromont@biomserv.univ-lyon1.fr 


\section{INTRODUCTION}

Classical Swine Fever (CSF) is a viral disease that affects wild and domestic swine worldwide. Outbreaks in domestic pigs entail severe losses to the pig farming industry because the virus is highly contagious and causes 20 to $90 \%$ mortality [12, 39]. Massive slaughtering is required to eradicate CSF and CSF-free countries restrict pig trading during outbreaks so that one outbreak in the Netherlands in 1997 caused more than $€ 2.5$ billion in losses [27]. Freeranging populations of European wild boars (Sus scrofa sp.) are regarded as potential reservoirs of CSF [24]. Since the CSF virus (CSFV) is able to survive in fomites and meat for several months [16], cross-contamination between wild boars and pigs may occur either through direct contact or through swill feeding of contaminated wild boars meat to commercial pigs [17]. Thus, the monitoring of CSF in populations of wild boars has become mandatory in the European Community. [9].

CSF monitoring can be performed on harvested wild boars by direct diagnosis, i.e., viral isolation [21] or indirect diagnosis, i.e., antibody counts [25]. Viroprevalence (or the proportion of viral positive animals) is generally used as an estimator of incidence $[10,21]$ and sanitary authorities generally declare the extinction of epizootics from the absence of virus isolation [24]. However, infected hosts may either die rapidly (within 10 to 30 days post infection in acute cases) or develop antibodies that neutralise the virus and offer lifetime protection [39]. Generally, the virus is not detected a few weeks post infection [39], so viroprevalence underestimates incidence at the level of the year [21]. Moreover, very young piglets that likely act as the infection reservoir $[13,21]$ are poorly sampled by hunting. Thus the viroprevalence we observe on hunted animals is only useful during the very early stages of epizootics, when instantaneous incidence peaks. Viroprevalence is not powerful enough to describe the evolu- tion of epizootics over the long term [10, $21]$, even when observed in large samples, which can be both time-consuming and expensive.

Seroprevalence (or a proportion of seropositive animals) reaches higher levels and is detected for longer periods of time than viroprevalence because antibodies are retained over the lifetime [25, 26, 39]. Overall, seroprevalence indicates the level of population immunity, because antibodies are protective $[12,39]$. Seropositive juveniles (except individuals less than three months in age that carry maternal antibodies $[12,13,26$, 39]) are of particular interest because they indicate a recent viral transmission. Thus, we propose that seroprevalence in juveniles gives an estimate of incidence at the level of the year that individuals were born. Since antibodies are retained over the lifetime of infected animals, seroprevalence is supposed to increase with age, except in the first year of the epizootic when all wild boars come in contact with the virus for the very first time [25, 34]. We thus consider, according to Laddomada et al. [25], that the difference in seroprevalence of a given cohort from year to year estimates incidence.

We analysed the spatial and temporal evolution of a CSF epizootic that occurred in northeastern France (northern Vosges Mountains) using mapping of seroprevalence in juveniles and temporal analysis of seroprevalence by age class. The first viral isolation was performed in early 1992. Previous inquiries, based on viroprevalence, suggested that CSF emerged in 1992 in the municipality of Philippsbourg [3, 10,31]. We used serological data to determine whether the French population was infected before 1992 and to pinpoint the location of the start of infection. After 1994, viroprevalence was very low $(<0.5 \%)$, and so did not provide detailed information on the spatial structure of incidence [10]. Lastly, no virus could be isolated after December 1997 [10,31]. Using serological data, we intended to describe the spatial and temporal evolution of incidence, and to test whether or not infection 
faded out after 1997 and whether CSF disappeared everywhere at the same time. CSF is expected to persist in large populations $[2,24]$. It is also expected to persist in a nonhomogeneous way, depending on factors favouring CSF transmission, such as local population dynamics or management measures [2, 24, 33, 37]. However, the detailed spatial pattern of CSF persistence has been poorly documented and we lack a theoretical understanding of the persistence of the disease [2].

\section{MATERIALS AND METHODS}

\subsection{Study area and sampling design}

The study area is located in the northern part of the Vosges Mountains in northeastern France ( $48^{\circ} 50^{\prime} \mathrm{N}$ and $7^{\circ} 30^{\prime} \mathrm{E}$ ) and covers $3030 \mathrm{~km}^{2}$, including $1180 \mathrm{~km}^{2}$ of woodland [10]. Motorways, a canal, rivers and towns constitute barriers to wildlife movements to the west, east and south of the area. The Vosges Forest is uninterrupted to the north and continues into Germany as the Pfalz Forest (Fig. 1).

French health authorities delineated two zones at the beginning of the CSF epizootic: the infected zone included all municipalities where positive viral isolation had been confirmed during the preceding six months, while the control zone constituted all remaining municipalities in the study area. The limit between the two zones changed over time according to the prevalence of virus isolation. The sampling plan used by the health authorities included all wild boars that were shot in the infected zone and at least $30 \%$ of the individuals that were shot in the control zone. The monitoring lasted from 1992 to 2002. However, no seropositive juvenile was detected after year 2000, thus we analysed the effects of age and space from 1992 to 2000 . Hunters sampled blood for serology and recorded the date, estimated age and municipality in a questionnaire for every wild boar shot. In 1991, around 5000 wild boars were shot in the whole survey area (source: Federation of hunters). Assuming that $40 \%$ to $50 \%$ of the population were shot every year $[5,18]$, we estimated the initial population size at 10000 to 12500 individuals.

\subsection{Determination of age classes and spatial locations}

Hunters classified each individual as a juvenile, yearling or adult, according to body mass and coat colour. The reddish browncoated individuals with a carcass weighing 10 to $30 \mathrm{~kg}$ were classified as juveniles, i.e., 5 to 12 months old. The black-coated animals with a carcass of 31 to $49 \mathrm{~kg}$ were classified as yearlings, i.e., 13 to 24 months old. Black-coated animals with a carcass heavier than $49 \mathrm{~kg}$ were classified as adults, i.e., aged more than 24 months old. This age classification was based on previous field studies of wild boars in northeastern France [22]. Animals less than three months old, i.e., striped and with a carcass less than $10 \mathrm{~kg}$, were not shot by hunters, so we assumed that there would be no maternal antibodies in the samples [12, 13, 26, 39]. Additionally, no vaccination of wild boars was implemented in France so we assumed that the wild virus had infected every seropositive animal.

The spatial location of wild boars was assigned to the $\left(\mathrm{x}_{\mathrm{i}}, \mathrm{y}_{\mathrm{i}}\right)$ coordinates of the municipality where they had been shot. We divided the time period from March 1992 to February 2001 into nine years, thereafter called seasons. Each season included one reproductive period (from March to September) and the entire hunting season (from October to February), according to the way local game experts record harvests.

\subsection{Diagnostic design}

A reference laboratory (AFSSA, Maisons-Alfort) searched blood samples for antibodies using a Neutralisation Peroxydase Linked Assay $[8,14]$. The laboratory 


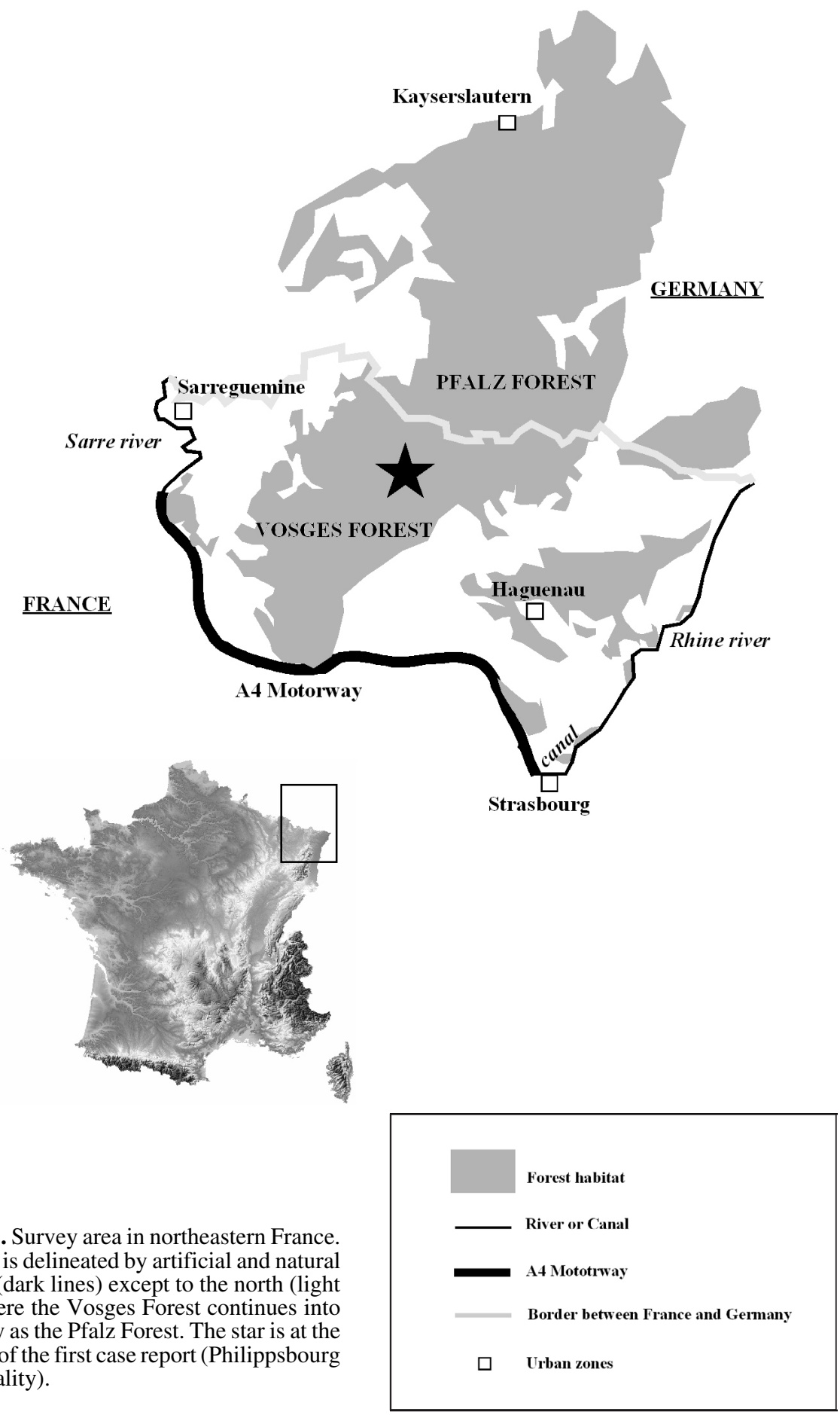

Figure 1. Survey area in northeastern France. The area is delineated by artificial and natural barriers (dark lines) except to the north (light line) where the Vosges Forest continues into Germany as the Pfalz Forest. The star is at the location of the first case report (Philippsbourg municipality). 
systematically performed differential diagnoses between CSFV and the closely related Border Disease virus (BDV) by comparing antibody titres for both viruses [14]. Seropositive cases included animals showing both a positive reaction at the $1 / 10$ dilution against CSFV and a higher titre for CSFV than BDV. This diagnostic process is the most sensitive and specific serological method currently available [14].

\subsection{Seroprevalence and estimate of CSF incidence}

We used seroprevalence to estimate annual incidence in each municipality. The epizootic was first detected in January 1992 in a susceptible population $[3,31]$. We first tested the hypothesis that the epidemic actually began in 1992 and so we expected that individuals of all ages should have been infected during 1992 and would show the same level of seroprevalence in 1992. We thus estimated incidence in 1992 using seroprevalence among all animals. After 1992, only antibodies in juveniles could demonstrate recent transmission of CSFV because antibodies are retained over lifetime [26, 39] so we then estimated incidence using seroprevalence among juveniles only.

Assuming that the observations were independent and that age classes were equally distributed over time and space, we calculated seroprevalence in each year and age class over the entire study area. We considered that seroprevalence at time $t$ estimated the proportion of animals that were infected at time $t$ or before this date. We also considered that $(1-$ seroprevalence at time $t)$ estimated the proportion of animals that were not yet infected by the virus at time $t$. We then considered that the probability for a yearling not to be infected at time $t$ (1 seroprevalence in yearlings at time $t$ ) was the cross product of the probability not to be infected at time $t-1(1-$ seroprevalence in juveniles at time $t-1)$ and of $(1-$ incidence in yearlings at time $t$ ). We used this hypothesis in order to estimate incidence in yearlings from 1993 to 2000 as [1 - (1 - seroprevalence in yearlings at time $t) /(1-$ seroprevalence in juveniles at time $t-1)]$. We calculated the variance of incidence in yearlings as the variance of the ratio of two independent variables [11].

\subsection{Variation of seroprevalence with age and time}

Our objective was to examine the temporal evolution of incidence and immunity in the different age classes and over the entire study area, in order to develop possible hypotheses on the mechanisms of disease persistence and extinction. We first plotted the evolution of the estimated incidence in juveniles and yearlings, as defined in Section 2.4.

Then we tested the effects of age and time on seroprevalence using logistic regression. We considered each individual separately so that we analysed seroprevalence as a binary variable. And we assumed that the observations were independent and that age classes were equally distributed over time and space. The effect of season was analysed either as a factorial $t$ or continuous $s$ variable. The effect of age was analysed as a factorial variable Age (juvenile, yearling, adult). We also tested the interaction between age and time. We calculated the Akaike criterion (AIC) for each possible model and retained the model with the lowest AIC value [1]. When the AIC difference between models was less than two, we retained the most parsimonious model, i.e., that with fewer parameters [1]. We analysed the goodness-of-fit of the final model using the Hosmer-Lemeshow test because of the binary nature of our dependent variable [15]. Then we tested difference among classes using the Wald tests [15].

\subsection{Analysis of spatial and temporal trends in incidence}

Our second objective was to analyse the temporal and spatial trends of incidence. Incidence was calculated for each year and 
each municipality and analysed using logistic regression. We analysed data grouped per municipality and per year, so that the number of observations per municipality and per year was employed as a weight of each observation. The effect of season was analysed either as a factorial $t$ or continuous $s$ variable. Contour maps of incidence were analysed using polynomial equations of the coordinates of the municipalities. We intentionally limited polynomial to the 3rd degree in order to get interpretable surfaces [23, 28]. We used the first-order polynomial to test for a planar trend surface, the secondorder polynomial to search for twists and the third-order polynomial to detect depressions and peaks $[23,28]$. We also included the interaction between temporal and spatial trends in order to determine whether the spatial structure of incidence changed over time. We used the same methodology as previously described for the comparison of models [1]. We used the Hosmer-Lemeshow goodness-of-fit test because we expected small sample size per municipality and per year [15].

The resulting polynomial model should comprise most of the spatial information on incidence, however, we tested for several structures in the residuals. We first tested spatial autocorrelation of residuals in order to detect potential second-order spatial effects. We defined municipalities as neighbours when the distance between their centroids was inferior or equal to $20 \mathrm{~km}$. We did not use classical definitions of neighbourhood such as the presence of a common border because the shape and surface of municipalities was highly variable, thus distant points might have been considered as neighbours whereas close points might not. Instead we used a definition of neighbours taking into account the biology of wild boars, because most wild boars do not disperse farther than $20 \mathrm{~km}$ from their birthplace [40]. To test for spatial autocorrelation, we used a Moran test that implemented 1000 permutations of the observed values of residuals [7]. We also tested whether the distributions of residuals across municipalities were correlated among years ("municipality effect" on residuals) using a Kruskall-Wallis test on municipalities that were sampled at least twice [35].

Statistical analyses were initially performed in R software [32]. We then depicted the predictions of the model using locally weighted regression [6] and ADE-4 software [38]. We compared incidence among municipalities calculating the predicted $95 \%$ confidence interval of each prediction. To give a graphical representation of the peak of incidence over years, we graphically represented the area including all municipalities where expected incidence was not significantly different from the municipality with maximal predicted incidence. This "area of highest incidence" was expected to include the center of the epidemic focus and to represent its spatial displacements over years.

\section{RESULTS}

\subsection{Description of the sample}

No seropositive juvenile was detected after the 2000 season, signifying that incidence was low or null in 2001. From 1992 to 2000,16799 wild boars were sampled, but blood samples could be analysed only for 8125 individuals because of bad sampling or conservation conditions for other sera. We removed animals of unknown age except in 1992, thus finally our analysis was performed on 6114 hunted animals, 38 of which were of unknown age, 3511 were classified as juveniles, 1732 as yearlings and 830 as adults (Tab. I). Serological data from 3674 animals were used to analyse the spatial and temporal variation of incidence, i.e., all animals sampled in 1992 and only juveniles thereafter. The number of municipalities sampled varied from 48 to 84 depending on the season, with a mean of 69 locations per year. Overall, the sample sizes varied across seasons from 132 to 653 individuals, with a mean of 408 per year. 
Table I. Number of seropositive wild boars and number of tested for each year and age class. Each cell gives the number of positive individuals followed by the number tested.

\begin{tabular}{cccc}
\hline Year & Juveniles & Yearlings & Adults \\
\hline 1992 & $44 / 99$ & $60 / 96$ & $14 / 29$ \\
1993 & $29 / 132$ & $31 / 44$ & $15 / 24$ \\
1994 & $91 / 385$ & $70 / 168$ & $85 / 144$ \\
1995 & $101 / 424$ & $76 / 230$ & $67 / 124$ \\
1996 & $63 / 380$ & $64 / 188$ & $55 / 108$ \\
1997 & $38 / 393$ & $49 / 178$ & $35 / 81$ \\
1998 & $52 / 513$ & $33 / 229$ & $28 / 95$ \\
1999 & $15 / 653$ & $12 / 292$ & $12 / 135$ \\
2000 & $4 / 532$ & $7 / 310$ & $7 / 90$ \\
Total & $437 / 3511$ & $402 / 1735$ & $318 / 830$ \\
\hline
\end{tabular}

\subsection{Variation of seroprevalence with age and time}

The best logistic model, regarding seroprevalence, included a significant effect of time $t$, as a factorial variable, of Age, and of the interaction $t \times A g e$ (Tab. II, Fig. 2). This model was well fitted to data according to the Hosmer-Lemeshow goodness-of-fit test (df $=7, P>0.999$ ), and took into account $20 \%$ of seroprevalence variability. The incidence estimated by seroprevalence in juveniles decreased over time. Significant broken down were observed between 1992 and $1993(\mathrm{OR}=0.352,[0.199 ; 0.623], P<$ $0.001), 1995$ and $1996(\mathrm{OR}=0.636,[0.447$; 0.903], $P=0.011), 1996$ and 1997 (OR = $0.539,[0.350 ; 0.828], P=0.005), 1998$ and $1999(\mathrm{OR}=0.208,[0.116 ; 0.375], P<$ $0.001)$, and 1999 and $2000(\mathrm{OR}=0.323$, [0.109; 0.957], $P=0.041)$.

Table II. AIC values of tested logistic models for CSF seroprevalence in wild boars. The retained model (in bold) included the effects of time $t$ and of age $A g e$ as factorial variables, and the interaction $t \times$ Age.

\begin{tabular}{lccc}
\hline Model & Degrees of freedom & Residual deviance & AIC \\
\hline Null & 6075 & 5916.00 & 5918.00 \\
Time as a linear variable $s$ & 6074 & 5129.85 & 5133.85 \\
Time as a factorial variable $t$ & 6067 & 5039.35 & 5057.35 \\
Age & 6073 & 5621.62 & 5627.62 \\
$t+$ Age & 6065 & 4763.83 & 4789.83 \\
$t \times$ Age & $\mathbf{6 0 4 9}$ & $\mathbf{4 7 3 0 . 0 9}$ & $\mathbf{4 7 8 4 . 0 9}$ \\
\hline
\end{tabular}

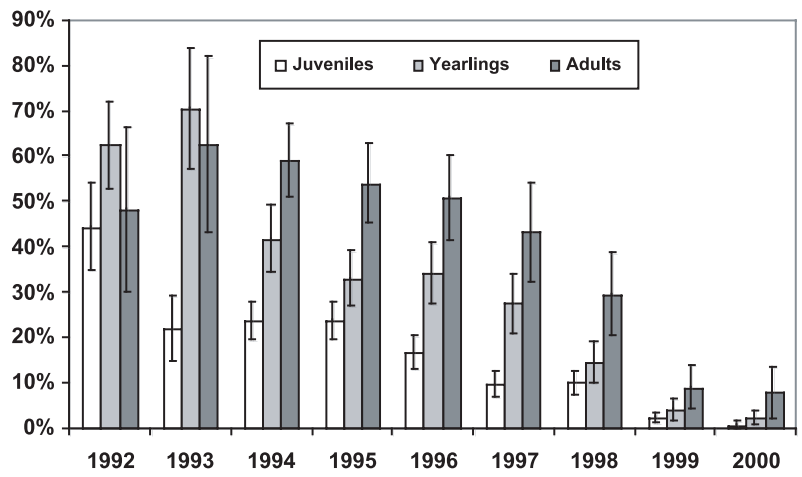

Figure 2. Predicted evolution of seroprevalence among three age classes (juveniles, yearlings, adults). Confidence bars represent $95 \%$ confidence intervals estimated by $1.96 \times$ (standard errors). 
Table III. Odds-ratios of seroprevalence between age classes from 1994 to 2000 and corresponding $P$-values of Wald tests. Odds-ratios are estimated by the exponential of the coefficients of the logistic model and significant Wald tests are in bold.

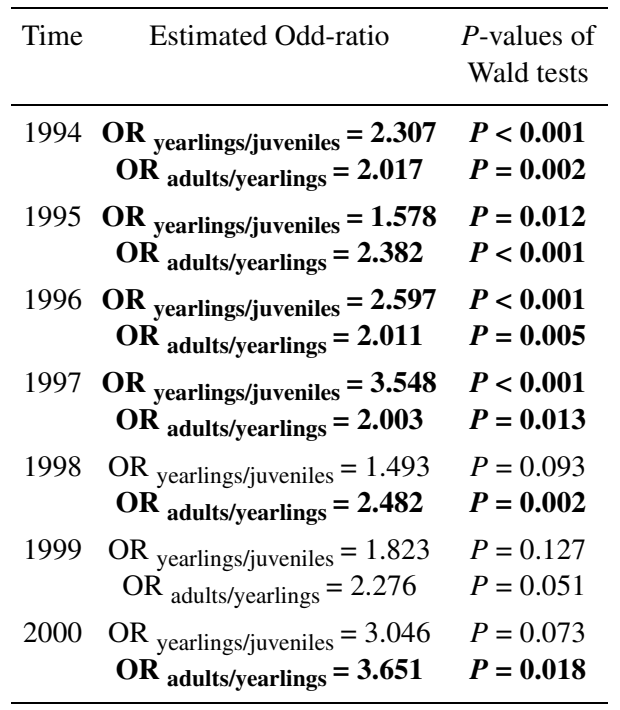

In 1992, we observed a higher seroprevalence in yearlings than in juveniles $(\mathrm{OR}=$ 2.083 , [1.175; 3.694], $P=0.012$ ), but we detected no difference between juveniles and adults $(P=0.716)$ nor between adults and yearlings $(P=0.175)$. In 1993 the seroprevalence was higher in yearlings and adults compared to juveniles $(\mathrm{OR}=8.083$,
$[3.922 ; 18.182], P<0.001)$ and was not significantly different between adults and yearlings $(P=0.504)$. From 1994 to 1997 , the gradual increase of seroprevalence with age was significant (Tab. III). After 1997, the gradual increase of seroprevalence with age was still observable but not always significant (Tab. III). Subsequently seroprevalence decreased in all age classes (Fig. 2), suggesting that both the proportion of newly infected individuals and overall population immunity decreased over time.

In yearlings, the estimated incidence followed a similar pattern as in juveniles, i.e., it decreased over time (Fig. 3). In 1992 and 1993, incidence was higher in yearlings than in juveniles, thus yearlings may have played an important role in disease propagation during this period. On the contrary, after 1998, estimated incidence was around zero, suggesting that the infection of yearlings was very scarce (Fig. 3).

\subsection{Analysis of the spatial and temporal trends of incidence}

The best model, regarding incidence, included the effect of season as a factorial variable, the second-order polynomial of the coordinates of the municipalities and the interaction between season and the spatial trend surface (Tab. IV). The HosmerLemeshow goodness-of-fit test was not significant ( $\mathrm{df}=6, P=0.163$ ), showing no

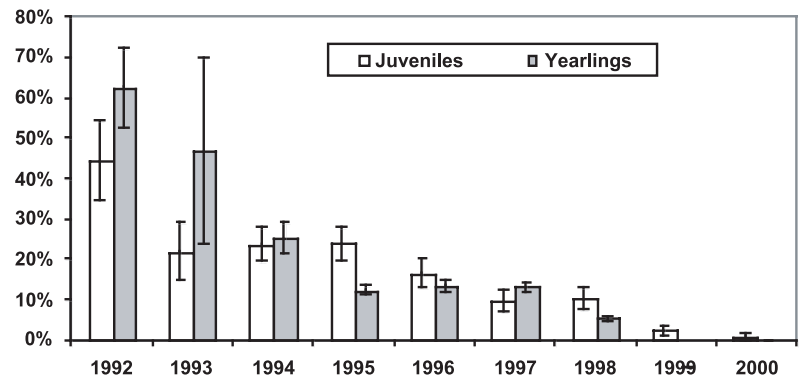

Figure 3. Evolution of incidence in juveniles and yearlings, estimated from seroprevalence data. Confidence bars represent $95 \%$ confidence intervals estimated by $1.96 \times$ (standard errors). 
Table IV. AIC values of tested logistic models for CSF incidence in wild boars. The retained model (in bold) included the effect of time $t$ as a factorial variable, the effect of space as a second-order trend surface and the interaction between time and space.

\begin{tabular}{lccc}
\hline Model & Degrees of freedom & Residual deviance & AIC \\
\hline Null & 617 & 1405.05 & 1407.05 \\
Time as a linear variable $\boldsymbol{s}$ & 616 & 895.41 & 899.41 \\
Time as a factorial variable $t$ & 609 & 836.32 & 856.32 \\
$t+$ linear polynomial $(\mathrm{x}, \mathrm{y})$ & 607 & 698.55 & 720.55 \\
$t+$ quadratic polynomial $(\mathrm{x}, \mathrm{y})$ & 604 & 555.97 & 583.97 \\
$t+$ cubic polynomial $(\mathrm{x}, \mathrm{y})$ & 600 & 551.43 & 587.43 \\
$t \times$ linear polynomial $(\mathrm{x}, \mathrm{y})$ & 591 & 668.13 & 722.13 \\
$\boldsymbol{t} \times$ quadratic polynomial $(\mathbf{x}, \mathbf{y})$ & $\mathbf{5 6 4}$ & $\mathbf{4 4 9 . 4 0}$ & $\mathbf{5 5 7 . 4 0}$ \\
$t \times$ cubic polynomial $(\mathrm{x}, \mathrm{y})$ & 528 & 383.90 & 563.90 \\
\hline
\end{tabular}

evidence that the model did not fit the data. The plot of squared normalised residuals (Fig. 4) shows no evidence of a spatial or temporal structure in residuals. Moreover $94 \%$ of the normalised residuals were between -2 and 2 , which was in accordance with the expected $5 \%$ under the hypothesis of a binomial distribution of the error. We found no significant spatial autocorrelation among residuals $(P>0.05$ each year), i.e., no second-order spatial effect was evident in the data. We also found no clear correlation among residuals in a given municipality at different dates (Kruskall-Wallis test $P=$ 0.067, df = 118).

On average, incidence decreased over time and also the spatial structure of incidence changed with time. Model predictions for each season are represented in Figure 5. Figure 6 gives the area of highest incidence. In 1992, our model predicts that the highest incidence was located in the Baerenthal municipality, in the northern area and near the supposed point of emergence (Philippsbourg municipality). However, the area of highest incidence also comprised many municipalities located in the northern area (Fig. 6), some of which being located along the border with Germany. In 1993, incidence was lower than incidence in 1992 in most of the survey area. From 1994 to 1999, incidence decreased in every part of the survey area (Fig. 5), but always remained the highest in the northern area (Fig. 6). In 2000 , incidence was very low and we observed no significant difference of incidence among municipalities.

\section{DISCUSSION}

\subsection{Does seroprevalence in juveniles correctly estimate incidence?}

We attempted to use seroprevalence in juveniles as an estimator of CSF incidence. Seroprevalence certainly underestimated the true incidence in juveniles, because they are particularly susceptible to CSFV, so that most of them would have died before developing antibodies [13, 39]. We suppose that this underestimation mainly affected the results in 1992, when virus-induced mortality was the highest [33]. Consequently, we also supposed that we overestimated incidence in yearlings in 1993. Since juveniles constitute the most susceptible class, we interpreted the absence of seropositive juveniles in 2001 as a break in the chain of transmission from 2000 to 2001 [36]. These findings highlight the reliability of serological compared to virological diagnoses: CSF transmission could be detected using observations of seropositive juveniles until 2000, 


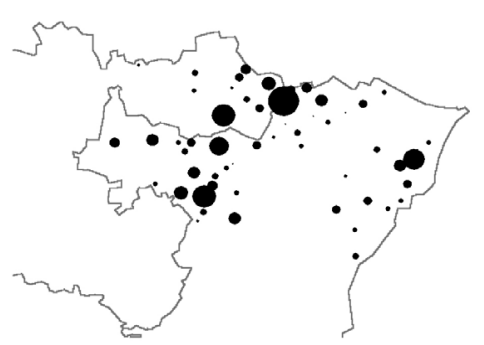

season 1992

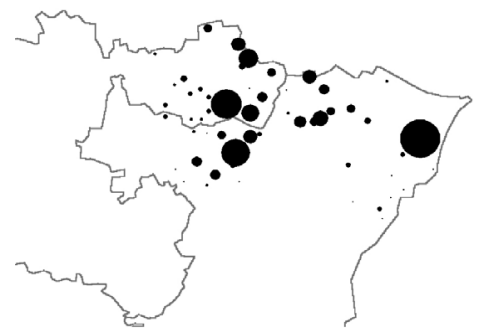

season 1995

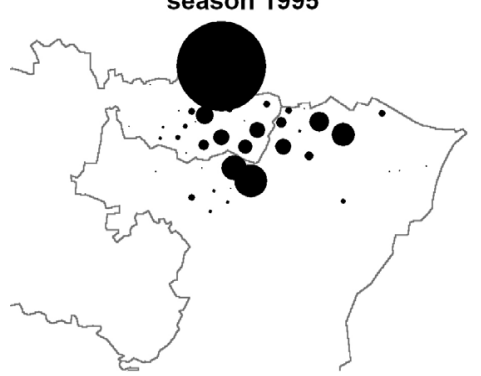

season 1998

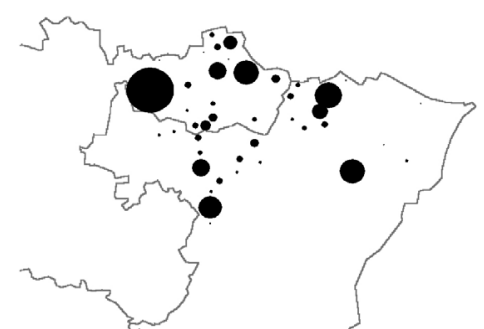

season 1993

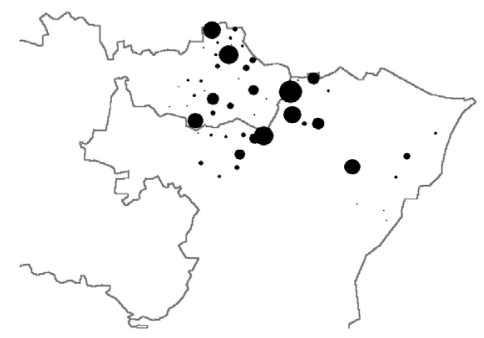

season 1996

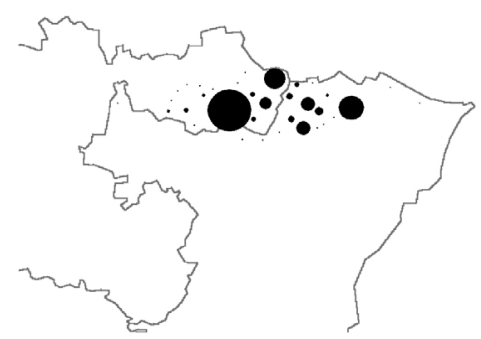

season 1999

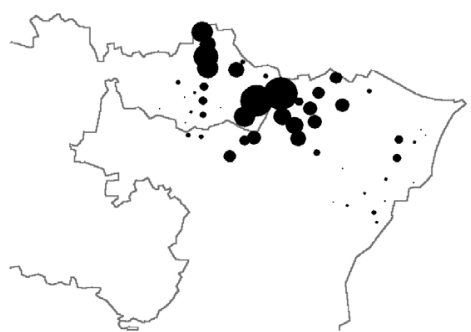

season 1994

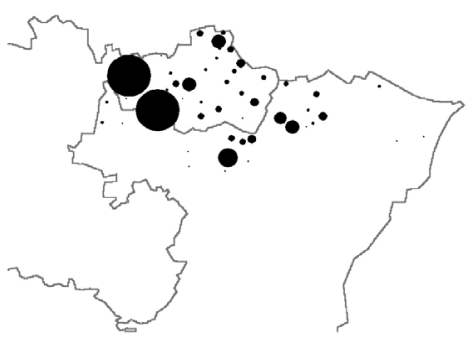

season 1997

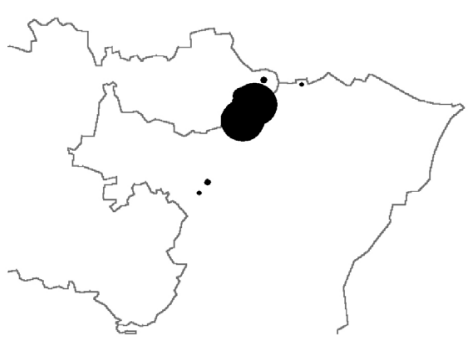

season 2000

Figure 4. Plot of the squared normalised residuals of the logistic model. 

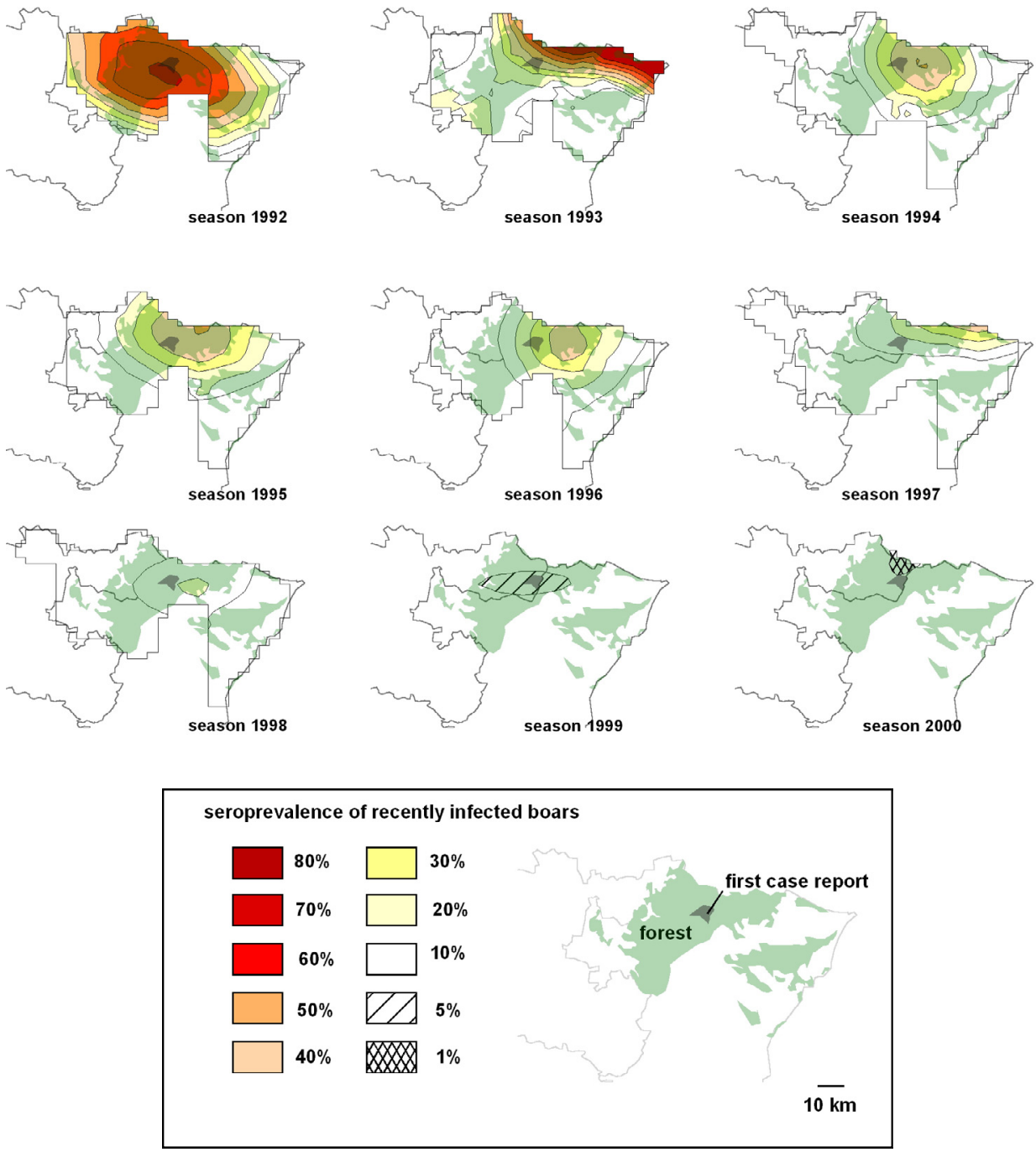

Figure 5. Incidence predicted from 1992 to 2000 using a model that included the effect of season, space (second-order polynomial) and the spatio-temporal interaction. Areas shaded in light-green represent the forested habitat and the area shaded in dark-grey represents the Philippsbourg municipality (see www.edpsciences.org/vetres for a colour version of this figure).

while no virus had been isolated since December 1997 [10]. Thus, we propose that the presence of seropositive juveniles in nonvaccinated populations should be implemented as the main criterion to determine the persistence of CSF infection. In the case of vaccinated populations, the antibody response of vaccinated and infected animals cannot be distinguished [39], so we propose that the presence of seropositive juveniles should be monitored, at least for one year (including the reproductive period) following the last vaccination campaign. The large sample of serological data allowed 


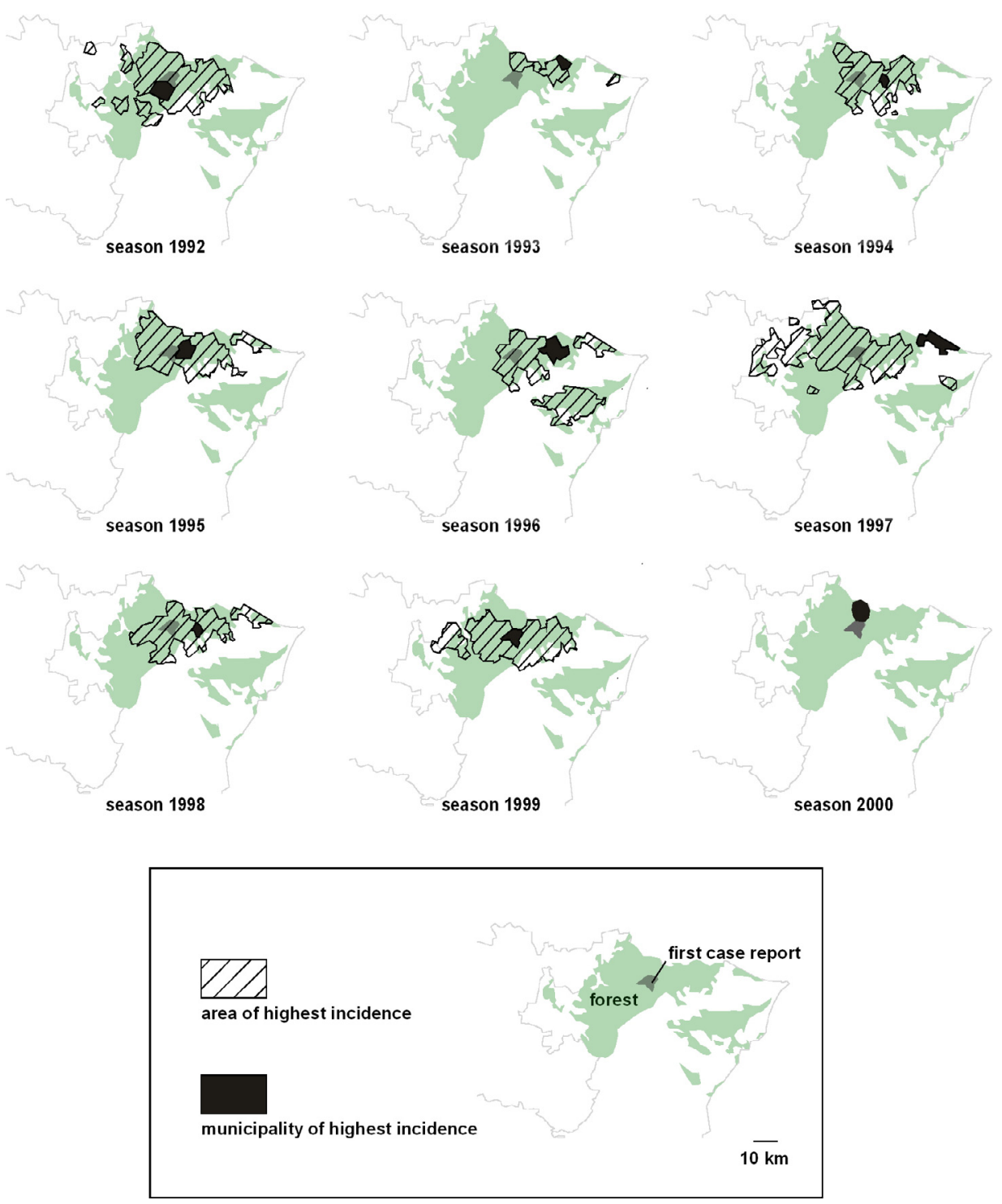

Figure 6. Predicted area of highest incidence ("peak area") from 1992 to 1999. Each year, the area in black corresponds to the municipality with maximal predicted incidence and the hatched area represents the contour of the municipalities where the predicted incidence was not significantly different from the highest incidence. Areas shaded in light-green represent the forested habitat and the area shaded in dark-grey represents the Philippsbourg municipality. In 2000, no significant difference was detected in the incidence among municipalities thus we did not define any area of maximal incidence (see www.edpsciences.org/vetres for a colour version of this figure). 
us to study the spatial structure of incidence over nine years while viroprevalence provided only three years of detailed spatial data $[3,10]$. These results encourage us to employ serological data for long-term monitoring of CSF in non-vaccinated populations.

\subsection{Reliability of incidence predictions}

The model we retained regarding the temporal and spatial evolution of incidence represented no more than $62.4 \%$ of the deviance of the null model. Thus, one must note that Figure 5 does not represent an exact prediction of incidence but rather a qualitative image of the evolution of incidence over space and time. Non-spatial factors, such as local population dynamics, structure of the habitat and local hunting habits, likely explain part of the residual variance in the data [33]. The HosmerLemeshow test did not show inadequacy in the goodness-of-fit and residuals revealed no particular structure, thus we had no indication of specific points to improve in the model.

\subsection{Hypotheses on emergence conditions}

In 1993 seroprevalence was not significantly different among yearlings and adults, but was inferior in juveniles compared to other age classes. Then, from 1994, we observed a gradual increase of seroprevalence with age. These results suggest that animals born in 1992 or before that year were equally exposed to CSF while after 1993 the risk was the highest in the oldest animals. In 1992, we observed a higher seroprevalence in yearlings than in juveniles but no difference between yearlings and adults or between juveniles and adults. We propose that the probable underestimation of incidence in juveniles in 1992 (see Sect. 4.1) may be responsible for the apparent difference between juveniles and yearlings. Given that antibodies are retained for the lifetime of infected animals, the pattern observed in
1992 and 1993 was consistent with the hypothesis that CSF emerged in 1992.

Polynomial modelling supports that incidence peaked near the supposed point of emergence, where the first virus isolation had been performed $[3,31,33]$. This observation suggests that CSF emerged in that part of the forest. However we observed no significant difference of incidence between the location of the peak and the municipalities located at the border with Germany; Baerenthal centroid is located at $10 \mathrm{~km}$ from the border, which is consistent with individual dispersal distances [40].

Because we cannot precisely locate the point of emergence, we propose two hypotheses for the cause of emergence of CSF in France. A first possibility is that wild boars consumed infected meat at feeding stations [24]. This may have arisen because hunters were not well informed on the sanitary risk of swill feeding in the early 1990's. Alternatively, CSF may have spread naturally from Reinland-Pfalz where virus isolation had been reported from 1991 on [24]. Although the second hypothesis is more parsimonious, we cannot choose between the two hypotheses since we only observed data in France.

\subsection{Hypothesis on the temporal and spatial variation in incidence}

Our model predicted that incidence peaked in the northern area and never reached the same peak values in the south. We observed the same pattern from virological data [33] and propose that, in the absence of a physical barrier within the Vosges forest, the apparent decrease of incidence over space may be due to differences in densities of wild boars, or to the low contact rate between wild boars of different social groups [33]. This observation is in accordance with predictions from mathematical models: when an epidemic occurs in a spatially structured population, several models predict that incidence should be the highest near the point of emergence and should decrease when the 
distance to the point of emergence increases $[4,29]$.

Although incidence was underestimated in 1992, our model predicted a significant decrease of incidence between 1992 and 1993 in most of the survey area. In 1993, the proportion of immune yearlings or adults was on average $60-70 \%$, and we thus propose that the massive immunisation of the population resulted in a decrease of virus transmission that year.

From 1993 to 2000, incidence decreased in the whole area. Two marked changes occurred after 1998: incidence decreased drastically and there was no evidence that infectious contact occurred in the yearlings. This second finding allowed us to propose a mechanism for extinction. Wild boars live in social groups comprised of adult females and juveniles for most of the year, with adult males roaming between groups [40]. Juveniles have contact with individuals in their natal group and thus participate in viral transmission within groups. Yearlings and adults also interact with other group members through dispersal or mating, and thus are probably responsible for most of the betweengroup transmission [40]. We assume that infection levels of yearlings and adults after 1998 (less than 10\%) were too low to have occurred between groups. If only certain social groups were infected, then only the litters in infected groups would develop antibodies. Infection faded out in 2000, which suggests that CSF cannot persist at the scale of isolated groups.

After 1993, CSF persisted only in the northeast of the survey area. Then, while infection seemed extinct in France after 2000, Rheinland-Pfalz health authorities declared viral isolations from 2001 to 2004 and infection re-emerged in France in April 2003 (Pacholek X., personal communication). These observations suggest that infection did not evolve independently on both sides of the borderline. This interdependence is supported by the fact that the forest cover is uninterrupted between France and Germany, and that displacement of wild boars across the border was confirmed by capture-mark-recapture [20]. So we can consider animals inhabiting the Vosges and Pfalz Forests as belonging to one single metapopulation. In this context, the most parsimonious explanation for CSF reemergence in 2003 is natural spreading from the Pfalz forest.

The longer persistence of infection in the northeast of our study area, and possibly in the Pfalz Forest, might be favoured by local conditions. This hypothesis is compatible with the enzootic situation of CSF in the Nuoro province of Sardinia, where the virus chronically infects surrounding areas [25, 30]. Contact between wild boars and domestic pigs are assumed to favour CSF persistence in Nuoro [24, 25, 30]. In our present case, conditions favourable to persistence could be a high host density, a high host birth rate or an optimal contact structure between the host social groups [33, 36, 37]. Additionally, we propose that infection persistence may be a dynamic phenomenon. Spatially structured stochastic mathematical models have demonstrated that infection can persist in a system of connected patches, even when persistence is not maintained in isolated patches [41]. According to this hypothesis, persistence over the long term is favoured by the large size of the forest and high number of social groups involved, both of which are consistent with theoretical findings of mathematical models and the empirical concept of critical host population size [19, 41].

We also observed a rapid loss of immunity in adults that we interpret as a consequence of the short lifespan of wild boars in the area, probably due to a high hunting pressure [18]. Such a high hunting pressure is also supposed to maintain a high birth rate in populations of wild boars through density-dependent mechanisms [33, 37]. We thus propose that a high hunting pressure may have favoured the maintenance of infection in the area, since healthy individuals may have rapidly replaced immune ones $[17,19,37]$. 
As a whole, the long-term dynamics of CSF in the area probably depends on conditions occurring in a larger area than that previously studied. Presently, information from both France and Germany is recorded in a common database, which should bring forth relevant information on the persistence of CSF.

\section{ACKNOWLEDGMENTS}

Data were collected by hunters, the Office National de la Chasse et de la Faune Sauvage, the Office National des Forêts, Veterinary Laboratories and Veterinary Offices from the Moselle and Bas-Rhin regions. We thank all those who performed fieldwork during the ten years. We also thank the reference laboratory (AFSSA, MaisonsAlfort) for performing the confirmation diagnosis and the French Ministry of Agriculture for funding the monitoring and epidemiological research (grant No. S00/41). We especially wish to thank D. Chessel, from UMR 5558, Université Claude Bernard Lyon 1, for contribution to the statistical and mapping processes and $\mathrm{H}$. Erb, from the Department of Population Medecine and Diagnostic sciences, NY State College of Veterinary Medecine, Cornell University, Ithaca, USA, for precious advice on previous version of the manuscript. Two anonymous referees also provided constructive comments. Finally, we thank W.J. King who reviewed the English of this paper.

\section{REFERENCES}

[1] Anderson D.R., Burnham K.P., White G.C., AIC model selection in overdispersed capture-recapture data, Ecology 75 (1994) 17801793.

[2] Artois M., Depner K.R., Guberti V., Hars J., Rossi S., Rutili D., Classical swine fever (hog cholera) in wild boar in Europe, Rev. Sci. Tech. Off. Int. Epizoot. 21 (2002) 281-303.

[3] Aubert M., Picard M., Fouquet E., Condé J., Crucière C., Ferry R., Albina E., La peste porcine classique du sanglier en Europe, Ann. Méd. Vét. 138 (1994) 239-247.

[4] Bailey N.T.J., The Mathematical theory of infectious diseases and its applications, 2nd ed., Charles Griffin \& Company LTD, London, 1975, pp. 175-185.

[5] Bouloire J.L., Vassant J., Le sanglier, Hatier, Paris, 1990.

[6] Cleaveland W.S., Delvin S.J., Locally weighted regression: an approach to regression analysis by local fitting, J. Am. Stat. Assoc. 83 (1988) 596-610.

[7] Cliff A.D., Ord J.K., Spatial processes: models and applications, Pion, London, 1981, pp. 63-65.

[8] Commission of the European Communities, Classical swine fever in wild boar, scientific committee on animal health and animal welfare, XXIV/B3/R09/1999, Brussels, 1999.

[9] Commission of the European Communities, Council Directive 2001/89/EC of the 23 October 2001 on Community measures for the control of classical swine fever, Off. J. Eur. Communities NL316 of 1.12.2001, 2001, pp. 5-35.

[10] Crucière C., Burger C., Gonzague M., Laboratory investigation of the "Massif Vosgien" CFS wild boar outbreak, in: European Commission doc (Ed.), IV/7196/98, Measures to control classical swine fever in European wild boar, Perugia, 1998, pp. 93-97.

[11] Dagnélie P., Théorie et méthodes statistiques, Vol. 1, 2nd ed., Les presses agronomiques de Gembloux, Gembloux, 1973.

[12] Dahle J., Liess B., A review on classical swine fever infections in pigs: epizootiology, clinical disease and pathology, Comp. Immunol. Microbiol. Infect. Dis. 15 (1992) 203-211.

[13] Depner K., Muller T., Lange E., Staubach C., Teuffert J., Transient classical swine fever virus infection in wild boar piglets partially protected by maternal antibodies, Dtsch. Tierarztl. Wochenschr. 107 (2000) 41-80.

[14] De Smit A.J., Laboratory diagnosis, epizootiology, and efficacy of marker vaccines in classical swine fever: a review, Vet. Q. 22 (2000) 181-188.

[15] Dohoo I., Martin W., Stryhn H., Veterinary epidemiologic research, AVC Inc., Charlottetown, Canada, $706 \mathrm{p}$.

[16] Edwars S., Survival and inactivation of classical swine fever virus, Vet. Microbiol. 73 (2000) 75-181.

[17] Fritzmeier J., Teuffert J., Greiser-Wilke I. Staubach C., Schluter H., Moennig V., Epidemiology of classical swine fever in Germany in the 1990's, Vet. Microbiol. 77 (2000) 29-41. 
[18] Gaillard J.M., Vassant J., Klein F., Some characteristics of the population dynamics of wild boar (Sus scrofa scrofa) in a hunted environment, Gibier Faune Sauvage 4 (1987) 31-47 (in French).

[19] Grenfell B., Harwood J., Metapopulation dynamics of infectious diseases, Trends Ecol. Evol. 12 (1997) 395-399.

[20] Hars J., Hamann J.L., Béfort J., Crucière C., Rossi S., Classical swine fever in Wild Boar in France. Results of a serological study of gruntlings captured in the Northern Vosges Mountain Range, in: Proc. 50th WDA Conference, Pilanesberg National Park, 2001, p. 222.

[21] Kern B., Depner K.R., Letz W., Rott M., Liess $B$., Incidence of classical swine fever (CSF) in wild boar in a densely populated area indicating CSF virus persistence as a mechanism for virus perpetuation, Zentralbl. Veterinarmed. 46 (1999) 63-67.

[22] Klein F., Contribution à l'étude de la croissance du sanglier (Sus scrofa) par capture et recapture, in: INRA Publ. (Ed.), Symposium international sur le sanglier, Toulouse, 1984, pp. 58-67.

[23] Krumbein W.C., Trend-surface analysis of contour-type maps with irregular control-point spacing, J. Geophys. Res. 64 (1954) 823-834.

[24] Laddomada A., Incidence and control of CSF in wild boar in Europe, Vet. Microbiol. 73 (2000) 121-130.

[25] Laddomada A., Patta C., Oggiano A., Caccia A., Ruiu A., Cossu P., Fininu A., Epidemiology of classical swine fever in Sardinia: a serological survey of wild boar and comparison with African swine fever, Vet. Rec. 134 (1994) 83-187.

[26] Matthaeus W., Korn G., Neutralizing antibodies in swine following the experimental infection with hog cholera virus, Zentralbl. Bakteriol. 204 (1967) 173-180 (in German).

[27] Meuwissen M.P.M., Horst H.S., Huirne R.B.M., Dijkhuizen A.A., A model to estimate the financial consequences of classical swine fever outbreaks: principals and outcomes, Prev. Vet. Med. 42 (1999) 249-270.

[28] Moore D.A., Spatial diffusion of raccoon rabies in Pennsylvania, USA, Prev. Vet. Med. 40 (1999) 19-32.

[29] Park A.W., Gubbins S., Gilligan C.A., Extinction time for closed epidemics: the effects of host spatial structure, Ecol. Lett. 5 (2002) $747-755$

[30] Patta C., Oggiano A., Cattina A., Vargiu M.P., Pala G., Melis P., Ladu A., Control of CSF in wild boar in Sardinia, in: European Commission doc (Ed.), IV/7196/98, Measures to control classical swine fever in European wild boar, Perugia, 1998, pp. 91-92.

[31] Picard M., Burger C., Plateau E., Crucière C., La peste porcine classique chez les sangliers : un visage épidémiologique nouveau de la maladie, Bull. Mens. Soc. Vet. Prat. Fr. 77 (1993) 81-97.

[32] R Development Core Team, R: A language and environment for statistical computing, $\mathrm{R}$ Foundation for Statistical Computing, Vienna, 2003.

[33] Rossi S., Fromont E., Pontier D., Crucière C., Hars J., Barrat J., Pacholek X., Artois M., Incidence and persistence of classical swine fever in free-ranging wild boar (Sus scrofa), Epidemiol. Infect. (in press).

[34] Schnyder M., Stark K.D.C., Vanzetti T., Salman M.D., Thur B., Schleiss W., Griot C., Epidemiology and control of an outbreak of classical swine fever in wild boar in Switzerland, Vet. Rec. 150 (2002) 102-109.

[35] Sokal R.R., Rolf F.J., Biometry, Freedma \& Co., New York, 1995

[36] Swinton J., Harwood J., Grenfell B.T., Harwood J., Persistence thresholds for phocine distemper virus infection in harbour seal Phoca vitulina metapopulations, J. Anim. Ecol. 67 (1998) 54-68.

[37] Swinton J., Woolhoose M.E.J., Dobson A., Ferroglio E., Guberti V., Grenfell B.T., Heesterbeek J.A.P., Microparasite transmission and persistence, in: Hudson P., Rizzoli A., Grenfell B., Heesterbeek H. (Eds.), Ecology of Wildlife Diseases, Oxford University press, Oxford, 2002, pp. 83-101.

[38] Thioulouse J., Chessel D., Doledec S., Olivier J.M., ADE-4: A multivariate analysis and geographical software, Statistics and Computing 7 (1996) 75-83.

[39] Van Oirschot J.T., Classical swine fever, in: Straw B.E., D'Allaire S., Taylor D.J., Mengeling W.L. (Eds.), Diseases Of Swine, 8th ed., Iowa State University Press, Ames, 1999, pp. 159172.

[40] Vassant J., Brandt S., Maillard D., Baubet E., Wild boar ethology and behaviour, in: European Commission doc (Ed.), IV/7196/98, Measures to control classical swine fever in European wild boar, Perugia, 1998, pp. 6-16.

[41] Wood S.N., Thomas M.B., Space, time and persistence of virulent pathogens, Proc. R. Soc. Lond. B Biol. Sci. 263 (1996) 673-680. 